\title{
Oscillon in Einstein-scalar system with double well potential and its properties.
}

\author{
Taishi Ikeda ${ }^{1, \star}$, Chul-Moon Yoo ${ }^{1}$, and Vitor Cardoso ${ }^{2,3}$ \\ ${ }^{1}$ Department of Physics, Graduate School of Science, Nagoya University, Nagoya 464-6602, Japan \\ ${ }^{2}$ CENTRA, Departamento de Física, Instituto Superior Técnico, Universidade de Lisboa, Avenida Rovisco \\ Pais 1, 1049 Lisboa, Portugal \\ ${ }^{3}$ Perimeter Institute for Theoretical Physics, 31 Caroline Street North Waterloo, Ontario N2L 2Y5, Canada
}

\begin{abstract}
The dynamical evolution of self-interacting scalar field has many nontrivial behaviors, which tell us many lessons in a nonlinear dynamics. On Minkowski spacetime, the scalar field with double well potential has localized, non-singular, time-dependent, long-lived solutions, which are called oscillons. The lifetime of the oscillon depends on the initial conditions. Furthermore, when the initial parameter is fine-tuned, oscillons can be infinitely, and type I critical behavior is observed. Here, we investigate the Einsteinscalar system with double well potential. We show that oscillons exist in this system, and discuss the behavior when the initial parameter is fine-tuned. Our results suggests that a new type of critical behavior appears in this theory.
\end{abstract}

\section{Introduction}

Nonlinear field equations often appear, and its nonlinearity plays an important role in many situations in physics. However, our understanding of the properties of the nonlinear effects is inadequate. In order to understand them more deeply, it is useful to focus on the universal feature of the nonlinear equations. Nonlinear scalar field equations in some classes have solitonic solutions, and it's formation, time evolution and stability are also important problems.

Here, we consider an Einstein-scalar system with a double well potential of the form

$$
V(\Phi)=\frac{\lambda}{4}\left(\Phi^{2}-\sigma^{2}\right)^{2}
$$

This theory has two interesting sources of non-linearity. One is the gravitational interaction, and the other is non-linear potential of the scalar field. When the gravity is turned off, there is localized, longevity, nonsingular solutions, which are called "oscillons", in this theory. On flat space, the oscillons have been studied by Bogolyubsky et al [1], and Copeland et al[2]. They found that the oscillons can be formed after a bubble collapses. The typical energy of the oscillon is about $43 / \sigma$, and the lifetime $\tau$ depends on the initial bubble radius. Typically, the lifetime is about $10^{2}-10^{3}\left(1 / \sqrt{\lambda \sigma^{2}}\right)$. However, when the initial bubble radius $R_{0}$ is fine-tuned to some value $R_{*}$, the lifetime obeys the scaling law:

$$
\tau=-\gamma \ln \left|R_{0}-R_{*}\right|+C
$$

^e-mail: ikeda@gravity.phys.nagoya-u.ac.jp 
where $\gamma$ and $C$ are constants. This behavior is similar to the type I critical behavior, which is known as universal feature of the gravitational system[3-6]. In this study, we investigate the effects of the gravity on the oscillon in the weak gravity case.

\section{Setting and numerical formulation}

\subsection{System}

We consider the Einstein-scalar theory described by the action

$$
S=\int d^{4} x \sqrt{-g}\left(\frac{R}{8 \pi G}-g^{\mu v} \Phi_{, \mu} \Phi_{, v}-2 V(\Phi)\right),
$$

where $V(\Phi)$ is the double well potential which is given by eq.(1). This system has a typical length scale $L=1 / \sqrt{\lambda \sigma^{2}} \cdot G \sigma^{2}$ is a dimensionless parameter which characterizes the strength of the gravitational interaction.

\section{2 formulation}

We solve the field equations numerically by using the generalized Baumgarte-Shapiro-ShibataNakamura (G-BSSN) formulation. G-BSSN formulation is a generalization of the BSSN formulation [7, 8] to the case of curvilinear coordinate.[9] This formulation is useful in spherically symmetric spacetime.

G-BSSN formulation is based on the $3+1$ decomposition and conformal decomposition. In the $3+1$ decomposition, the line element is given by

$$
d s^{2}=-\alpha^{2} d t^{2}+\gamma_{i j}\left(d x^{i}+\beta^{i} d t\right)\left(d x^{j}+\beta^{j} d t\right)
$$

where $\alpha$ is a lapse function, $\beta^{i}$ is a shift vector, and $\gamma_{i j}$ is a 3 -metric. Extrinsic curvature $K_{i j} \equiv$ $\left(\frac{\partial}{\partial t}-\mathcal{L}_{\beta}\right) \gamma_{i j}$ is also important quantity. In the conformal decomposition, the 3 metric and the extrinsic curvature are decomposed to $\phi, \tilde{\gamma}_{i j}, K, \tilde{A}_{i j}$, which are defined by

$$
\begin{gathered}
\gamma_{i j}=e^{4 \phi} \tilde{\gamma}_{i j}, \\
K_{i j}=e^{4 \phi} \tilde{A}_{i j}+\frac{1}{3} \gamma_{i j} K .
\end{gathered}
$$

The determinant of $\tilde{\gamma}_{i j}$ is given from the determinant of the reference metric $\bar{\gamma}_{i j}$. Furthermore, for the numerical stability, we introduce the auxiliary field:

$$
\tilde{\Lambda}^{k} \equiv \tilde{\gamma}^{i j}\left(\tilde{\Gamma}_{i j}^{k}-\bar{\Gamma}_{i j}^{k}\right)=\tilde{\gamma}^{i j} \Delta \tilde{\Gamma}_{i j}^{k}
$$

In the case of the spherically symmetric spacetime, the nontrivial components of the $\tilde{\gamma}_{i j}, \tilde{A}_{i j}, \beta^{i}, \tilde{\Lambda}$ are as follows:

$$
\begin{gathered}
\tilde{\gamma}_{i j}=\operatorname{diag}\left(a, b r^{2}, b r^{2} \sin ^{2} \theta\right) \\
\tilde{A}_{i j}=\operatorname{diag}\left(A, B r^{2}, B r^{2} \sin ^{2} \theta\right), \\
\beta^{i}=(\beta, 0,0), \\
\tilde{\Lambda}^{i}=(\tilde{\Lambda}, 0,0) .
\end{gathered}
$$

We can get the time evolution equations for $a, b, \phi, A, B, K, \tilde{\Lambda}, \Phi$ and $\Pi$ which is a momentum conjugate of the scalar field (see [11]). 


\section{3 gauge condition}

We use the harmonic gauge condition:

$$
\partial_{t} \alpha=\mathcal{L}_{\beta} \alpha-K \alpha^{2}
$$

and $\beta=0$.

\section{4 numerics}

We use the iterative Crank-Nicolson scheme [12] for the integration in time, and a 2nd order finite difference method for spatial derivative. In order that the solutions remain smooth, we add the KreissOliger dissipation.

\section{5 boundary condition}

For numerical simulation, it is necessary to impose the boundary conditions. For the regularity at the origin, we must impose the Neumann boundary conditions,

$$
\begin{aligned}
& \left.\alpha^{\prime}\right|_{t=0}=\left.\phi^{\prime}\right|_{r=0}=\left.K^{\prime}\right|_{r=0}=\left.\tilde{\Lambda}\right|_{r=0} \\
& =\left.\Phi^{\prime}\right|_{r=0}=\left.\Pi^{\prime}\right|_{r=0}=0, \\
& \left.a^{\prime}\right|_{r=0}=\left.b^{\prime}\right|_{r=0}=\left.A^{\prime}\right|_{r=0}=\left.B^{\prime}\right|_{r=0}=0 \text {. }
\end{aligned}
$$

For the local flatness at the origin, we must impose the Dirichlet boundary condition for $a, b, A, B$ :

$$
\left.a\right|_{r=0}-1=\left.b\right|_{r=0}-1=\left.A\right|_{r=0}=\left.B\right|_{r=0}=0 .
$$

If this Dirichlet boundary condition is satisfied on the initial data, the evolution equations guarantee this boundary condition at any time. However, for the numerical simulation, this condition can be violated, and the violations is the origin of the numerical instability. In this study, we explicitly impose the Neumann boundary condition for $\alpha, \phi, K, \tilde{\Lambda}, \Phi, \Pi$ and the Dirichlet boundary condition for $a, b, A, B$. The outer boundary condition is the asymptotically Minkowski spacetime.

\section{6 definition of the lifetime}

We define the lifetime $\tau$ of the oscillon as follows:

$$
\frac{M\left(\tau ; r_{0}\right)}{M\left(0 ; r_{0}\right)}=\epsilon \ll 1,
$$

where $M() \tau ; r_{0}$ is a Kodama mass inside a sphere of the radius $r_{0}[13,14]$.

\subsection{Initial data}

In this study, we use momentary static Gaussian bubble and a spatially conformally flat initial data:

$$
\begin{gathered}
\Phi(t=0, r)=-\sigma+2 \sigma e^{-r^{2} / R_{0}^{2}} \\
a(t=0, r)=b(t=0, r)=1,
\end{gathered}
$$

where $R_{0}$ is a radius of the bubble. 

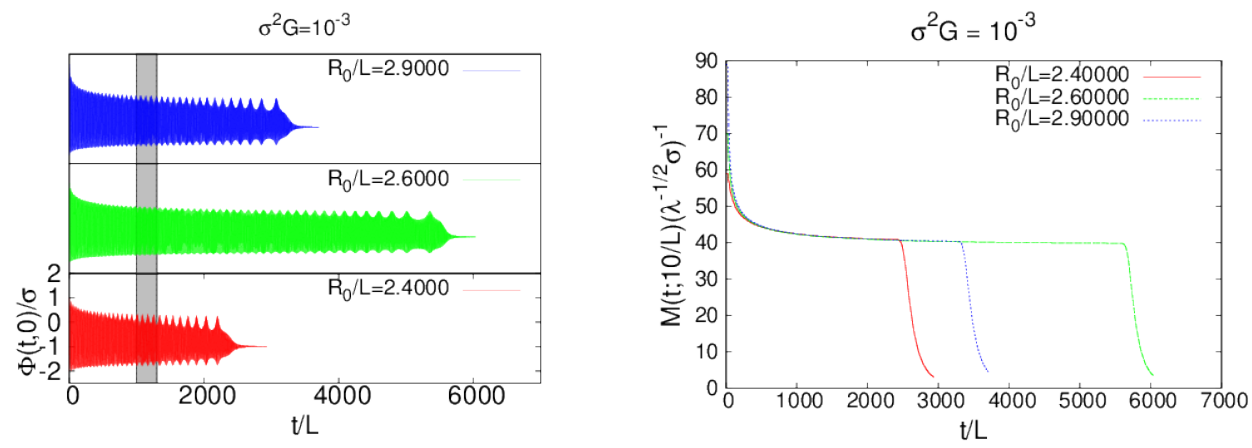

Figure 1. Left panel shows envelope of the time evolution of $\Phi(t, r=0)$ for $\sigma^{2} G=1.0 \times 10^{-3}$. Right panel show the time evolution of the Kodama mass.

\section{Results}

This system has one model parameter $\sigma^{2} G$ which characterize the strength of the coupling between the scalar field and gravity. In this study, we study the oscillon's formation and it's property for $\sigma^{2} G=1.0 \times 10^{-4}, 5.0 \times 10^{-4}, 1.0 \times 10^{-3}$ and $2.0 \times 10^{-3}$.

We find that the self-gravitating oscillon appears after the bubble collapses, and its typical behavior is similar to the case of flat background(see Fig.1) Oscillon's typical energy depends on $\sigma^{2} G$ (see Table.1). Furthermore, when the bubble radius is fine-tuned, the type I critical behavior appears for

Table 1. The dependence of $M_{\text {Oscillon }}$ on $\sigma^{2} G$.

\begin{tabular}{|c|c|}
\hline$\sigma^{2} G$ & $M_{\text {Oscillon }} / L$ \\
\hline $1.0 \times 10^{-4}$ & 43 \\
\hline $5.0 \times 10^{-4}$ & 41 \\
\hline $1.0 \times 10^{-3}$ & 40 \\
\hline $2.0 \times 10^{-3}$ & 38 \\
\hline
\end{tabular}

$\sigma^{2} G=1.0 \times 10^{-4}, 5.0 \times 10^{-4}, 1.0 \times 10^{-3}$, that is $\tau$ obeys the scaling low:

$$
\frac{\tau}{L}=-\gamma \ln \left|\frac{R_{0}-R_{*}}{L}\right|+C
$$

For $\sigma^{2} G=2.0 \times 10^{-3}, \tau$ shows periodic modulations around the scaling (see Fig.2):

$$
A \cos \left(-\log \left|\frac{R_{0}-R_{*}}{L}\right|+\varphi\right) \equiv \frac{\tau}{L}-\left(-\gamma \log \left|\frac{R_{0}-R_{*}}{L}\right|\right)-\delta .
$$

This behavior may be new type of the critical behavior.

\section{References}

[1] I. L. Bogolyubsky and V. G. Makhankov, JETP Lett. 24, 12 (1976). 

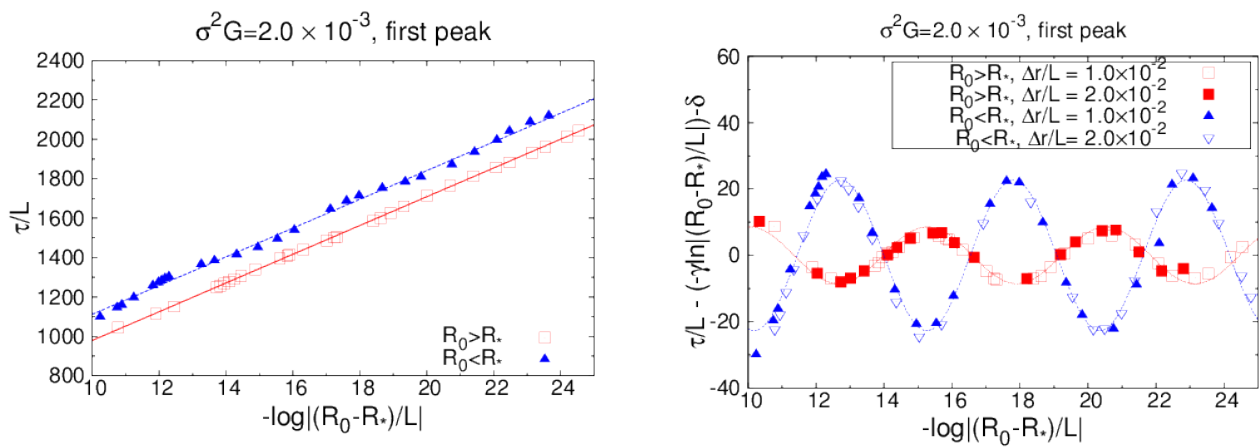

Figure 2. The left panels show the relation between the lifetime $\tau$ and the initial bubble radius near the first three peaks for $\sigma^{2} G=2.0 \times 10^{-3}$. The right panel shows the deviation between the lifetime as predicted by scaling law and our actual numerical results. We checked the convergence of our result.

[2] E. J. Copeland, M. Gleiser and H.-R. Muller, Phys. Rev. D 52, 1920 (1995) doi:10.1103/PhysRevD.52.1920 [hep-ph/9503217].

[3] M. W. Choptuik, Phys. Rev. Lett. 70, 9 (1993). doi:10.1103/PhysRevLett.70.9

[4] C. Gundlach and J. M. Martin-Garcia, Living Rev. Rel. 10, 5 (2007) doi:10.12942/lrr-2007-5 [arXiv:0711.4620 [gr-qc]].

[5] K. Clough and E. A. Lim, arXiv:1602.02568 [gr-qc].

[6] T. Ikeda and C. M. Yoo, Phys. Rev. D 94, no. 12, 124032 (2016) doi:10.1103/PhysRevD.94.124032 [arXiv:1610.07280 [gr-qc]].

[7] M. Shibata and T. Nakamura, Phys. Rev. D 52, 5428 (1995). doi:10.1103/PhysRevD.52.5428

[8] T. W. Baumgarte and S. L. Shapiro, Phys. Rev. D 59, 024007 (1999) doi:10.1103/PhysRevD.59.024007 [gr-qc/9810065].

[9] J. D. Brown, Phys. Rev. D 79, 104029 (2009) doi:10.1103/PhysRevD.79.104029 [arXiv:0902.3652 [gr-qc]].

[10] A. Akbarian and M. W. Choptuik, Phys. Rev. D 92, no. 8, 084037 (2015) doi:10.1103/PhysRevD.92.084037 [arXiv:1508.01614 [gr-qc]].

[11] T. Ikeda, C. M. Yoo and V. Cardoso, arXiv:1708.01344 [gr-qc].

[12] S. A. Teukolsky, Phys. Rev. D 61, 087501 (2000) doi:10.1103/PhysRevD.61.087501 [grqc/9909026].

[13] H. Kodama, Prog. Theor. Phys. 63, 1217 (1980). doi:10.1143/PTP.63.1217

[14] T. Harada, C. M. Yoo, T. Nakama and Y. Koga, Phys. Rev. D 91, no. 8, 084057 (2015) doi:10.1103/PhysRevD.91.084057 [arXiv:1503.03934 [gr-qc]]. 